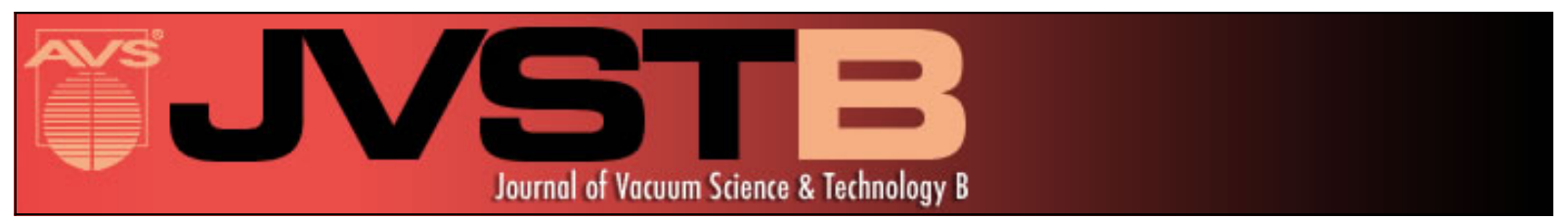

\title{
Simultaneous detection of positive and negative secondary ions
}

Richard J. Chater, Alan J. Smith, and Graham Cooke

Citation: Journal of Vacuum Science \& Technology B 34, 03H122 (2016); doi: 10.1116/1.4943531

View online: http://dx.doi.org/10.1116/1.4943531

View Table of Contents: http://scitation.aip.org/content/avs/journal/jvstb/34/3?ver=pdfcov

Published by the AVS: Science \& Technology of Materials, Interfaces, and Processing

\section{Articles you may be interested in}

Positive and negative ion formation in deep-core excited molecules: $\mathrm{S} 1 \mathrm{~s}$ excitation in dimethyl sulfoxide

J. Chem. Phys. 140, 024314 (2014); 10.1063/1.4861050

Simultaneous direct detection of sub keV molecular and atomic ions with a delta-doped charge-coupled device at the focal plane of a miniature mass spectrometer

Appl. Phys. Lett. 88, 043501 (2006); 10.1063/1.2166701

Quadrature detection for the separation of the signals of positive and negative ions in fourier transform ion cyclotron resonance mass spectrometry

AIP Conf. Proc. 606, 647 (2002); 10.1063/1.1454343

Multimass ion imaging detection: Application to photodissociation

Rev. Sci. Instrum. 72, 1963 (2001); 10.1063/1.1359188

Development of a triplasmatron ion source for the generation of SF $5+$ and $\mathrm{F}$ - primary ion beams on an ion microscope secondary ion mass spectrometry instrument

J. Vac. Sci. Technol. A 17, 845 (1999); 10.1116/1.581657

\section{Instruments for Advanced Science} w www.HidenAnalytical.com E info@hiden.co.uk CLICK TO VIEW our product catalogue

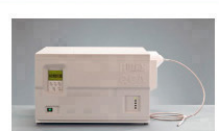

Gas Analysis

dynamic measurement of reaction gas streans catalysis and thermal analysis molecular beam studies

species probes

, fermentation environmental and ecological studies

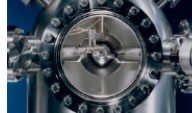

Surface Science

UHVTPD

SIMS emental imaging - surface mapping

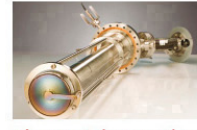

Plasma Diagnostics plasma source characterization etch and deposition process reaction. , analysis of neutral and radical species

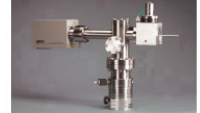

Vacuum Analysis , partial pressure measurement and control of process gases reactive sputter process control vacuum diagnostics renum coating processmonitoring 


\title{
Simultaneous detection of positive and negative secondary ions
}

\author{
Richard J. Chater ${ }^{\text {a) }}$ \\ Department of Materials, Imperial College of Science, Technology and Medicine, London SW7 2AZ, \\ United Kingdom
}

Alan J. Smith and Graham Cooke

Hiden Analytical Ltd, Warrington WA5 7UN, United Kingdom

(Received 14 December 2015; accepted 26 February 2016; published 9 March 2016)

\begin{abstract}
A secondary ion mass spectrometer (SIMS) instrument is described that is configured with two SIMS detectors that are both low-field extraction, quadrupole-based filters. Secondary ions are generated by sputtering with a liquid-metal ion gallium source and column of the type that is common on twobeam electron microscopes. The gallium ion beam, or focused ion beam achieves sub- $100 \mathrm{~nm}$ focus with a continuous current of up to $300 \mathrm{pA}$. Positive secondary ions are detected by one SIMS detector, and simultaneously, negative secondary ions are detected by the second SIMS detector. The SIMS detectors are independently controlled for recording mass spectra, concentration depth profiles, and secondary ion images. Examples of simultaneous positive and negative SIMS are included that demonstrate the advantage of this facility for surface analysis and depth profiling. The SIMS secondary ion collection has been modeled using the ray tracing program SIMION ("SIMION", Scientific Instrument Services, Inc., Ringoes, NJ, 08551-1054, see http://www.simion.com) in order to understand the interaction of the secondary ions of opposite polarities in the extraction volume for the purpose of optimizing secondary ion collection. (C) 2016 American Vacuum Society.
\end{abstract}

[http://dx.doi.org/10.1116/1.4943531]

\section{INTRODUCTION}

Secondary ion mass spectrometry (SIMS) is an analytical technique for surface chemical analysis by sputtering the target material with a primary ion beam and detection in a vacuum of the emitted secondary ions on a mass to charge $(\mathrm{m} / \mathrm{z})$ ratio basis. The radio frequency electric quadrupole ${ }^{1}$ is a very convenient method of filtering secondary ions that is combined with a secondary electron multiplier (SEM) for single particle detection. The combination can provide almost $100 \%$ duty cycle. Many material surfaces of interest have contamination or corroded surface layers with complex chemical composition containing compounds of metal and nonmetals with functional grouping such as oxides, carbonates, chlorides, etc. These surface layers or isolated features provide the focus of interest in many studies such as oxidation and corrosion as they determine the chemical nature of the surface, for example, localized corrosion of stainless steel. ${ }^{2}$ The secondary ion sputtered flux will contain both positive and negative particles whatever the primary species. Generally, oxygen or cesium primary ions are chosen as these elements enhance the yield of electropositive and electronegative elements, respectively, reducing the influence of the surface chemistry on the secondary ion yield. Access to the analysis of both electropositive and electronegative elements $(\mathrm{M})$ can be achieved with sputtering by cesium beams and the detection of $\mathrm{MCs}^{+}$or $\mathrm{MCs}_{2}{ }^{+}$molecular secondary ions although their yield is low relative to the enhanced elemental ion yields. ${ }^{3}$ For cesium sputtering, the pre-steadystate region prior to steady state erosion is very dependent on the sample and instrument configuration. ${ }^{4}$ If the primary ion etching is by an inert gas ion, the chemical nature of the

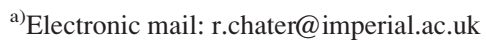

surface layer is relatively unaltered apart from the physical effects such as ion-beam damage, preferential sputtering, enhanced diffusivity, etc., whether or not the inert gas atoms are incorporated into the matrix. ${ }^{5}$ In this context, the use of gallium as a primary ion is very comparable to inert gas ions as secondary ion yields are not enhanced as with oxygen or cesium. $^{4}$

\section{SIMULTANEOUS POSITIVE AND NEGATIVE SECONDARY ION DETECTION}

As the basis of the SIMS analysis process is a destructive analysis, the advantages of simultaneous capture of both positive and negative ions with the potential for a high duty cycle is demonstrated in this paper with two quadrupolebased SIMS detectors and a gallium primary ion source and column that has the potential for sub-100 nm lateral resolution. Hayashi ${ }^{6}$ has demonstrated a design for simultaneous positive and negative (SPN) with a single quadrupole $\mathrm{m} / \mathrm{z}$ filter and low-energy $(<10 \mathrm{eV})$ charged ion separation at the exit of the filter with a separate SEM for secondary ions of each polarity. There is no polarization of the collection field at the entrance to the quadrupole, so positive and negative ions of the same $\mathrm{m} / \mathrm{z}$ ratio are filtered simultaneously without charge bias. The main disadvantages are that it would not be possible with this SPN design for detection of two different mass ions and a diminished sensitivity due to the lack of an extraction field for enhanced secondary ion detection. The absence of a secondary ion energy filter at the entrance to the quadrupole allows high energy neutrals and fast sputtered and backscattered ions to pass through the quadrupole unanalyzed. ${ }^{7}$ The effect is a substantial background countrate even with the SEM detector off-axis. Daolio ${ }^{8}$ reported a novel SPN design in which a newly designed quadrupole 
electrostatic analyzer/deflector was built for secondary ion separation by both energy and polarity. In common with Hayashi, there is no low-field extraction for secondary ions. Secondary ions emitted at near-normal angles $\left(\theta<10^{\circ}\right)$ and entering the electrostatic analyzer/deflector are subsequently filtered by separate quadrupole systems for SPN detection, potentially of different mass ions. The design details given ${ }^{8}$ indicate that the entrance aperture of $2 \mathrm{~mm}$ diameter is between 5 and $6 \mathrm{~mm}$ from the target. The results from a molybdenum test sample sputtered in a pressure of $5 \times 10^{-6}$ mbar of oxygen show a very low background count-rate that is less than 10 counts/s which, at that pressure, is 2 orders of magnitude lower than that reported by Wittmaack. ${ }^{7}$ The effect of the electrostatic analyzer in the SIMS detector configuration used in this paper has been reported ${ }^{9}$ with similar sputtering and pressure conditions, giving a background count-rate of less than 1 counts/s. The background to maximum peak count-rate of up to 8 orders of magnitude provides a significant advantage of quadrupole-based and magnetic sector systems compared with time-of-flight mass spectrometers. ${ }^{10}$ In this context, it is worth mentioning that a configuration using a single quadrupole detector and secondary ion polarity switching, including the extraction field, would suffer from a lateral shift in the sputtered area for the two polarities that is dependent on the primary beam energy. At the primary ion beam energy of $30 \mathrm{keV}$ required for sub$100 \mathrm{~nm}$ beam focus, the lateral shift is several microns.

\section{INSTRUMENT CONFIGURATION}

A single beam gallium ion microscope, FEI FIB200 workstation ${ }^{11}$ with a quadrupole-based SIMS detector has been fitted with a second SIMS detector, a Hiden EQS $1000,{ }^{12}$ which is also quadrupole-based but is separately and differentially pumped. ${ }^{9}$ The relative orientation of the detectors is shown in Fig. 1 with respect to the impact point of the primary ion beam on the sample target. The height position referred to as the eucentric height in Fig. 1 is reproducible to less than $5 \mu \mathrm{m}$ from sample to sample and at different impact sites on each sample. In the notation of Fig. 1(b), the eucentric height of a feature is determined by rotating the feature through $45^{\circ}$ of stage tilt about the $\mathrm{Y}$-axis and choosing the $\mathrm{Z}$-coordinate value at which the feature remains at the center of the primary beam scanned area. At this position, the distance between the exit aperture of the primary beam column and the feature surface (working distance) is $19 \mathrm{~mm}$. The configuration of the SIMS detectors shown in Fig. 1 does not compromise any of the specifications of the FEI FIB200 workstation. The beam ion energy and current are independent of the sample orientation. The geometrical collection angle for the two detectors is between $1 \%$ and $2 \%$ of $2 \pi$ steradians. Both detectors have separate low-field extraction for the secondary ions of both polarities which enhance the collection angle for secondary ions. This enhanced collection efficiency is in part due to the nonlinear, $\cos (\theta)$ form of the secondary ion emission envelope ${ }^{13}$ where $\theta$ is the angle to the target normal, and which is particularly appropriate for a conducting polycrystalline oxide target (see Sec. IV A) that

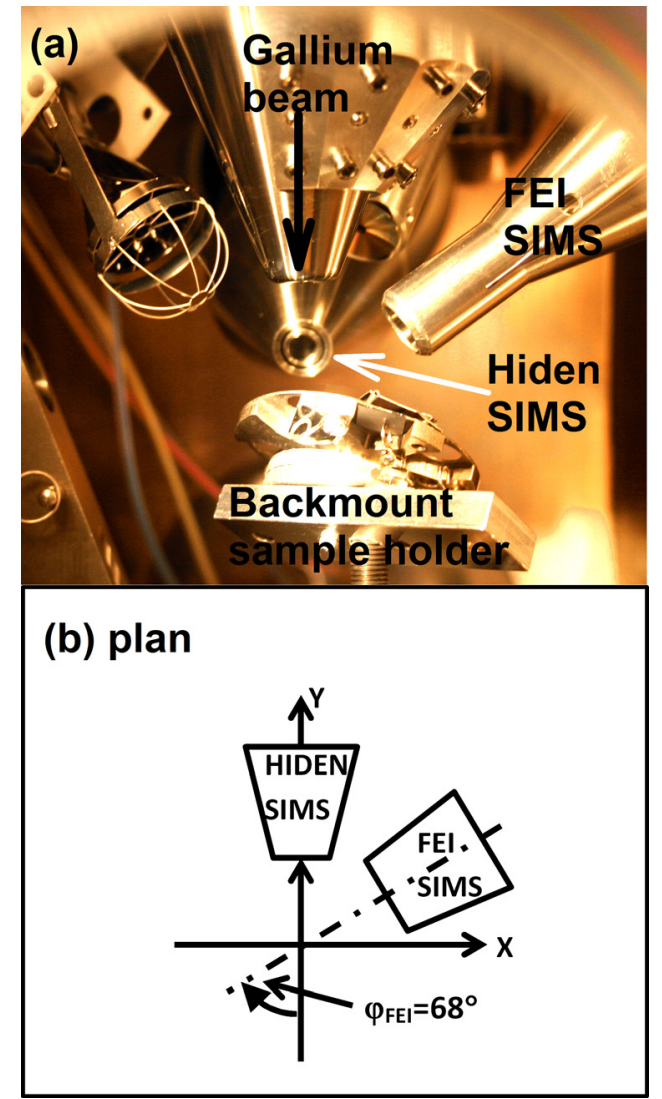

FIG. 1. (Color online) Configuration of the two SIMS detectors, gallium primary ion column, and back-mount sample holder is shown in the photograph (a) and the schematic plan (b). The X, Y directions are the axes of the sample stage, and the origin is the gallium primary ion impact point at the eucentric height on the $\mathrm{Z}$-axis that is perpendicular to the $\mathrm{XY}$ plane through the origin. The distances from the origin to the front of the FEI detector is $18 \mathrm{~mm}$ and to the Hiden detector is $15 \mathrm{~mm}$. The tilt angles for the two detectors seen in (a) above the XY plane are $\alpha_{\text {Hiden }}=19^{\circ}$ and $\alpha_{\mathrm{FEI}}=35^{\circ}$.

amorphizes during the SIMS process. Figure 1(b) schematic indicates that the axes of the SIMS detectors are aligned through the impact point of the primary ion beam at eucentric height. This is a simplification as the detector axes are slightly offset where the estimated distance between the axis and the impact point is between 1 and $2 \mathrm{~mm}$ at the closest point.

\section{RESULTS}

\section{A. Pre-steady-state region and preferential sputtering for the LSM oxide target}

Gallium ion sputtering at $7 \mathrm{keV}$ of the conducting polycrystalline oxide, lanthanum strontium manganate $\left(\mathrm{La}_{0.8} \mathrm{Sr}_{0.2} \mathrm{MnO}_{3}\right)$ or LSM oxide target, is estimated by SRIM (Ref. 14) to have an ion range of $5.3 \mathrm{~nm}$ and a straggle of $2.6 \mathrm{~nm}$. In Fig. 2(b), the gallium positive secondary ion yield due to the resputtered implanted gallium ion, ${ }^{69} \mathrm{Ga}^{+}$, achieves a steady state yield after $600 \mathrm{~s}$ of sputtering with a primary ion dose density (PIDD) of $4 \times 10^{14}$ gallium ions per $\mathrm{cm}^{2}$ and this initial time period is called the pre-steadystate region. The molecular ion ${ }^{155}(\mathrm{LaO})^{+}$achieves a steady state level after the same period of $600 \mathrm{~s}$ of sputtering. In 


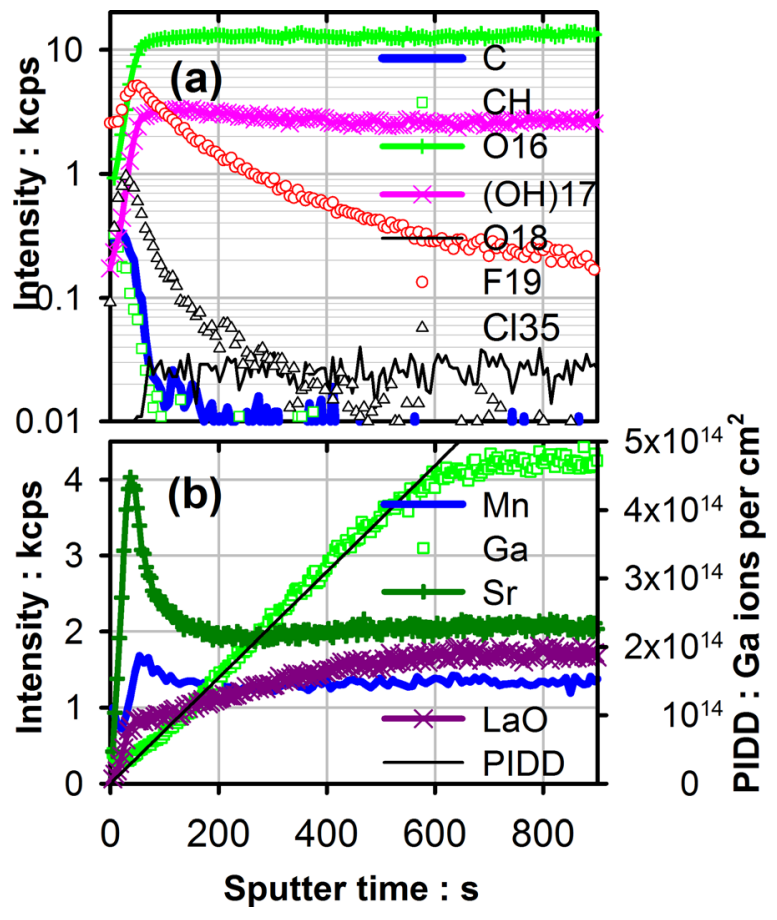

FIG. 2. (Color online) Pre-steady-state region for the LSM oxide target sputtered by gallium ion for the negative secondary ions (a) and (b) the simultaneously detected positive secondary ions. (b) also includes the gallium PIDD from the sputtering conditions, see text.

contrast both the atomic ions ${ }^{55} \mathrm{Mn}^{+}$and ${ }^{88} \mathrm{Sr}^{+}$achieve steady state sputtering after a much shorter period of $200 \mathrm{~s}$ and a sputter dose of $1.6 \times 10^{14}$ gallium ions per $\mathrm{cm}^{2}$. The oxide surface is covered with a thin hydrocarbon contamination layer indicated by the profiles for the negative ions, ${ }^{12} \mathrm{C}^{-}$and ${ }^{13} \mathrm{CH}^{-}$, removed after $80 \mathrm{~s}$ of sputtering. Both ions have short linear decays on the log-linear plot in Fig. 2(a). Sputter removal of the contamination layer reveals the oxide matrix when the negative atomic oxygen ions ${ }^{16} \mathrm{O}^{-}$and ${ }^{18} \mathrm{O}^{-}$achieve steady state sputtering after only $80 \mathrm{~s}$ of sputtering and a dose $6.4 \times 10^{13}$ gallium primary ions per $\mathrm{cm}^{2}$, i.e., the PIDD for the pre-steady-state period is shorter for oxygen by almost a tenth of time required for the ${ }^{69} \mathrm{Ga}^{+}$or the ${ }^{155}(\mathrm{LaO})^{+}$ions.

The isotopic fraction of oxygen-18 is calculated from the intensities of the ${ }^{16} \mathrm{O}^{-}$and ${ }^{18} \mathrm{O}^{-}$secondary ions as $(0.0020 \pm 0.0004)$ at each point in the profile after $80 \mathrm{~s}$ from the start of sputtering. This value is to be compared to the natural abundance for oxygen-18, which is very similar at $(0.00204 \pm 0.00001){ }^{15}$ The PIDD for each point in the profile is $5.6 \times 10^{12}$ gallium primary ions per $\mathrm{cm}^{2}$, which is within the static SIMS dose limit. The $17\left(\mathrm{OH}^{-}\right)$negative ion is steady throughout the profile once the oxygen matrix of the LSM oxide is revealed and is assumed therefore to be associated with the proton content of the oxide, which might be expected depending on the thermal anneal history of the sample. Both the ${ }^{19} \mathrm{~F}^{-}$and ${ }^{35} \mathrm{Cl}^{-}$ions have profiles in the pre-steady-state region that are very different to the carbon ions of the contamination layer. In particular, the fluorine surface peak is a substantial fraction of the oxygen-16 intensity, indicating that the fluorine is probably at matrix

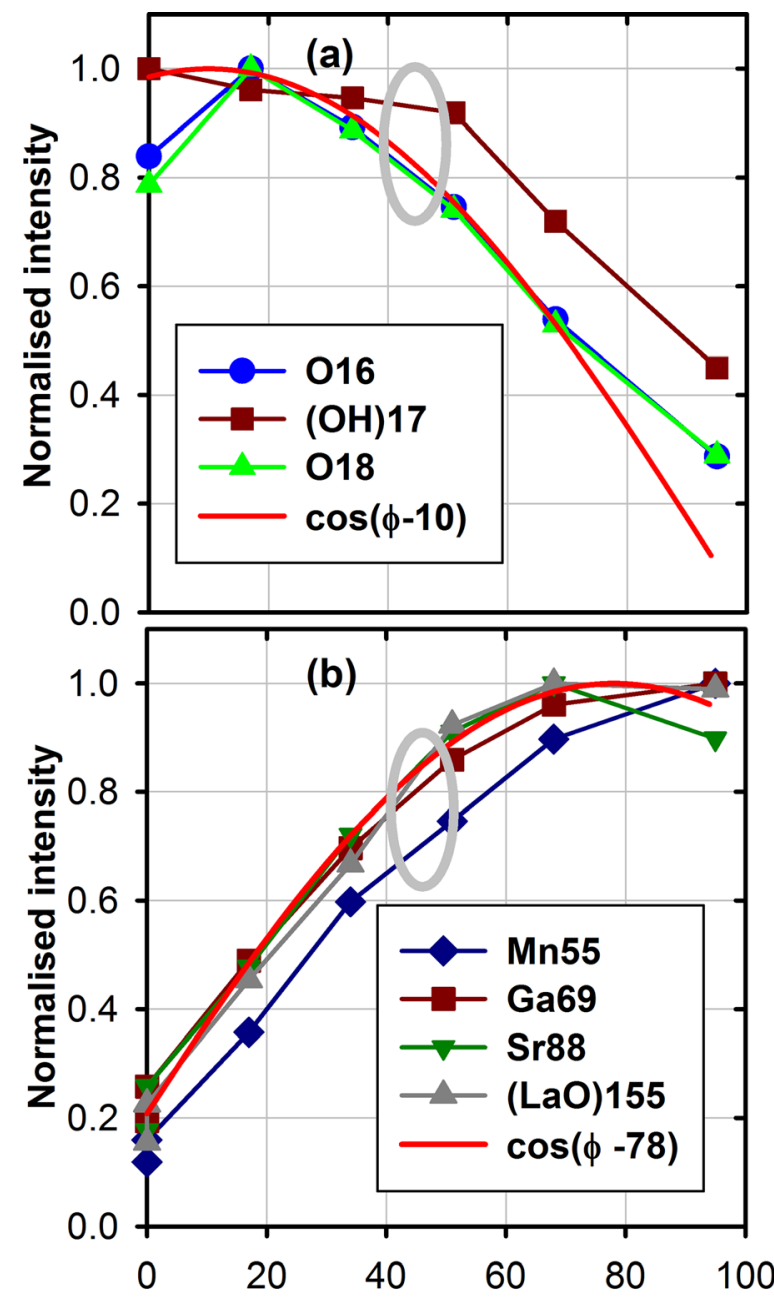

Rotation angle, $\phi$ : degrees

FIG. 3. (Color online) Variation in the negative (a) and positive (b) secondary ion intensities as the LSM oxide target surface is rotated to positions between the two SIMS detector directions. A rotation, $\phi=0^{\circ}$, is the position when the sample is facing the negative ion detector (Hiden). The sample faces the positive ion detector (FEI) at angle, $\phi_{\mathrm{FEI}}=68^{\circ}$. Gray ellipse highlights angle of $\phi=40^{\circ}-50^{\circ}$ where both normalized intensities are matched at 0.8 . Primary beam is incident at an angle, $\theta=30^{\circ}$ to the target surface normal. Measurement at symbol positions, lines between symbols are for eye guidance only. $\operatorname{Cos}(\phi)$ plotted in each pane with $\phi$ differing by the configuration constant of $68^{\circ}$, see text.

concentrations and occupying oxygen lattice sites. ${ }^{16} \mathrm{It}$ is important to recall that the background residual gas vacuum contributes to the intensities of the oxygen, hydroxyl, and fluorine negative ion intensities shown in Fig. 2(a).

Oxygen is a "volatile" component that is expected to be preferentially sputtered whereas lanthanum is the least volatile as it has the highest surface binding energy (SBE) of $4.46 \mathrm{eV} .{ }^{17}$ Both the manganese and strontium have intermediate SBE values, 2.9 and $1.7 \mathrm{eV}$, respectively, so it was expected that these ions would show a different pre-steadystate profile, indicating that the surface chemistry in the sputter crater has a dominant effect. The SPN depth profile gives a very full picture benefitting from both polarities. The duty cycle for each positive ion is $25 \%$ and for each negative ion was $14 \%$ in the depth profile of Fig. 2. With 11 secondary 


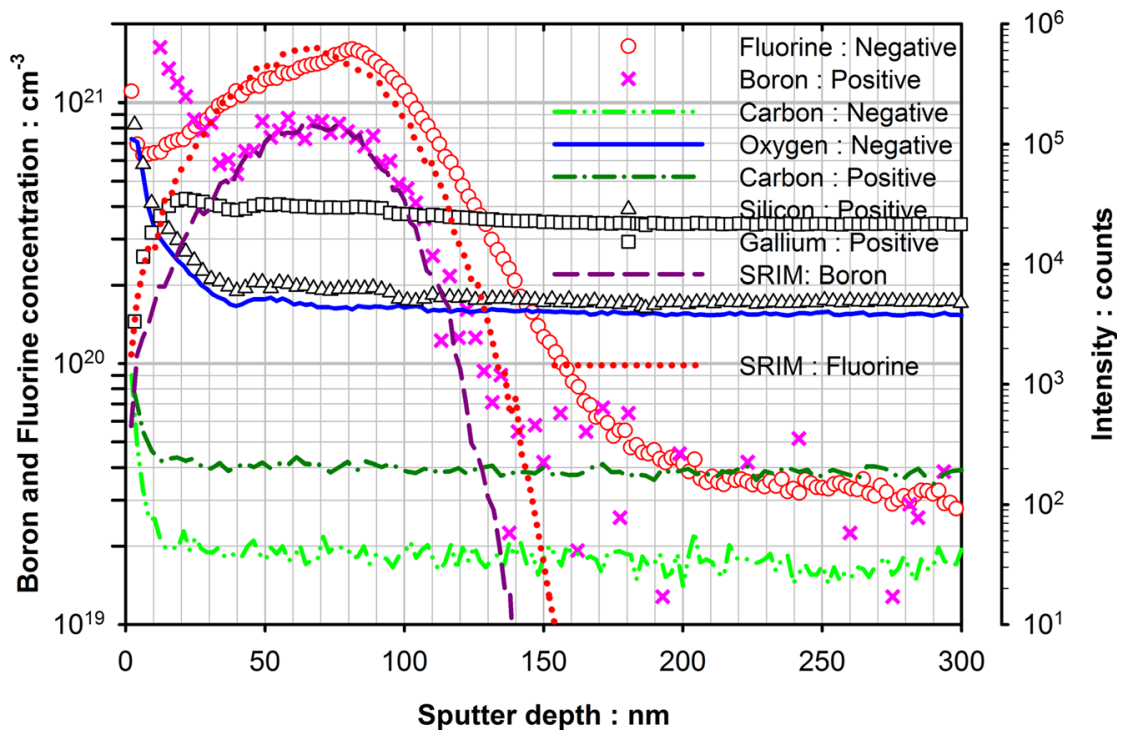

FIG. 4. (Color online) SPN SIMS analysis of $\mathrm{BF}_{2}{ }^{+}$implant into silicon at $70 \mathrm{keV}$ for ${ }^{11} \mathrm{~B}^{+}$and ${ }^{19} \mathrm{~F}^{-}$depth profiles. The implanted species have been concentration calibrated using a SRIM estimation and the specified implant dose of $6 \times 10^{15} \mathrm{BF}_{2}{ }^{+}$ions per $\mathrm{cm}^{2}$. No depth shift was applied to the sRIM simulated profiles for boron and fluorine.

ions recorded in the depth profile, this performance compares well with the available magnetic sector or time-offlight SIMS instrumentation. ${ }^{10}$

\section{B. Optimization of sample orientation}

The LSM oxide target was sputtered with the gallium primary ions at $30 \mathrm{keV}$ and $3.28 \mathrm{nA}$, and the normalized intensities of a number of the positive $\left({ }^{55} \mathrm{Mn}^{+},{ }^{69} \mathrm{Ga}^{+},{ }^{88} \mathrm{Sr}^{+}\right.$, and $\left.{ }^{155} \mathrm{LaO}^{+}\right)$and negative $\left[{ }^{16} \mathrm{O}^{-},{ }^{17}\left(\mathrm{OH}^{-}\right),{ }^{18} \mathrm{O}^{-}\right]$atomic and molecular secondary ions were recorded by SPN SIMS in the steady state sputtering regime. The variation in the secondary ion intensities as the LSM oxide target is rotated $(\varphi)$ between the two detectors is shown in Figs. 3(a) and 3(b) for a number of rotation angles with the expectation that the intensity variation is continuous. For these results, the LSM oxide target surface was fixed at an angle, $\theta=30^{\circ}$, to the normal with the gallium primary ion beam. The graphs in Fig. 3 show normalized yields of the selected atomic and molecular matrix secondary ions as the sample holder is rotated away from facing the Hiden SIMS detector at a rotation angle of $\varphi=0^{\circ}$. Both plots include the $\cos (\theta)$ form of the secondary ion emission envelope where the configurational angle of $68^{\circ}$ between the two detectors is maintained. The optimum plot position for the $\cos (\theta)$ envelope was chosen by eye and included a ten degree offset, the origin of which is unknown. The shape of the measured intensity with sample rotation followed the $\cos (\theta)$ form for LSM matrix ions. Figure 3 shows that there is a compromise to be made with simultaneous use of the two SIMS detectors. A matched intensity reduction factor of 0.8 for both SIMS detectors is achieved for a rotation of the target between $\varphi=40^{\circ}$ and $50^{\circ}$. The actual rotation angle $\phi$, chosen for any analysis, is not fixed but will reflect the objectives of the analysis and the available SIMS yields.

\section{Silicon ( $\mathrm{BF}_{2}$ implant)}

The SIMS analysis of this implant sample using SPN SIMS detection is shown as an example of semiconductor SIMS analysis requirements. In this silicon semiconductor sample, the ${ }^{49}\left(\mathrm{BF}_{2}\right)^{+}$ions at $70 \mathrm{keV}$ implanted into silicon are detected simultaneously as ${ }^{11} \mathrm{~B}^{+}$and ${ }^{19} \mathrm{~F}^{-}$in the depth profile (Fig. 4). A number of other matrix $\left({ }^{28} \mathrm{Si}^{+}\right)$, beam $\left({ }^{69} \mathrm{Ga}^{+}\right)$, and surface specific $\left({ }^{12} \mathrm{C}^{+},{ }^{12} \mathrm{C}^{-},{ }^{16} \mathrm{O}^{-}\right)$secondary ions are also profiled. Figure 4 shows the surface carbon contamination and native oxide layers are mixed into the substrate. The boron and fluorine concentration calibrations are based on the estimates of peak concentration from the SRIM simulations of the implant which are also plotted in Fig. 4. The depth calibration of the profile assumes a uniform sputter rate. The surface positive ion enhancements for boron and silicon finish where the ${ }^{16} \mathrm{O}^{-}$ion intensity becomes uniform at a depth of $40 \mathrm{~nm}$. No attempt was made to estimate the boron enhancement in the surface oxide/contamination layer or the associated sputter-rate change. The surface contamination layer and native oxide layer are judged to be very thin based on the correspondence between the measured and simulated boron profiles despite the broadening associated with the primary ion beam. In contrast, the comparison of the measured and simulated fluorine distribution demonstrates radiation-enhanced diffusion during sputtering. The gallium primary ions at $30 \mathrm{keV}$ used for this sputter depth profile have a SRIM (Ref. 14) estimated range and straggle in silicon of 27 and $9 \mathrm{~nm}$, respectively.

\section{RAY-TRACING MODELING IN SIMION}

An optimized configuration for SPN with two Hiden SIMS detectors, Fig. 5(a), is modeled by SIMION (Ref. 18) for ray tracing of secondary ions sputtered by a normally incident gallium primary beam. The SPN detection is shown by the trajectories of negative sputtered ions and positive 

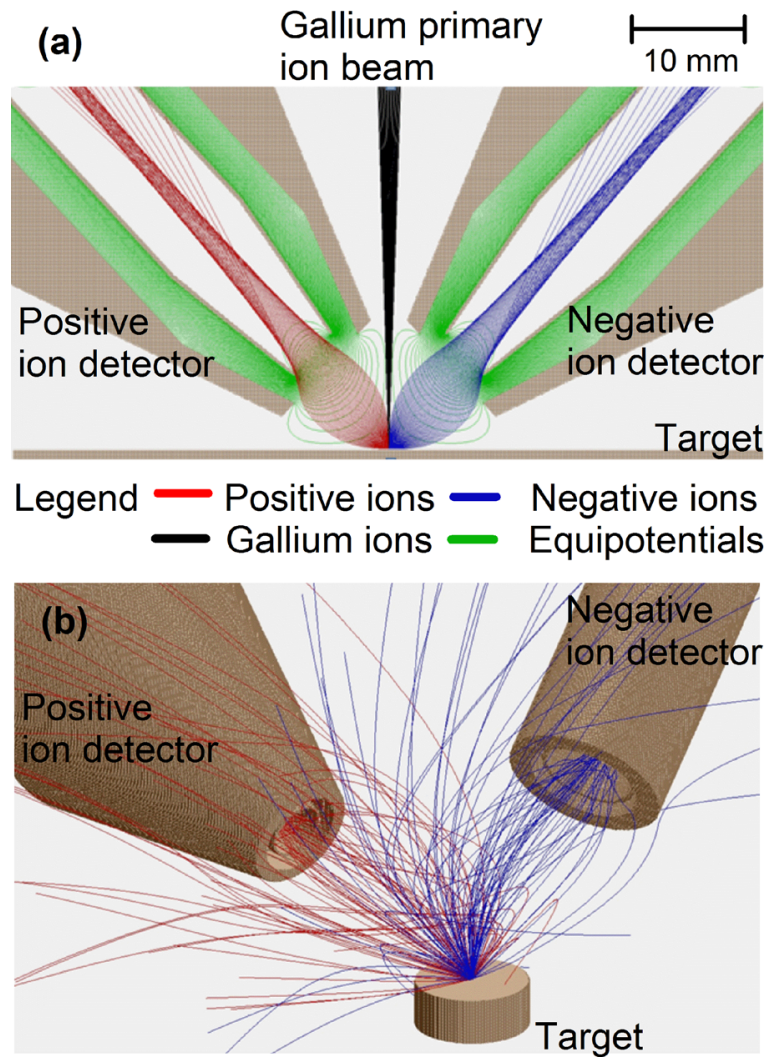

FIG. 5. (Color online) (a) SIMION 3D (Ref. 18) ray-tracing model of an optimized configuration for SPN secondary ion detection with two Hiden detectors and secondary ions sputtered by a normally incident gallium primary beam. (b) SIMION 3D (Ref. 18) ray-tracing model of the actual configuration shown in Fig. 1 with normal incidence gallium ions.

sputtered ions from the sample target into the appropriate SIMS detector. Only the paths of the secondary ions that have an angular distribution of $\pm 45^{\circ}$ about each detector axis are shown. The sample bias is $0 \mathrm{~V}$, and the initial secondary ion energy is $5 \mathrm{eV}$ with $100 \mathrm{~V}$ extraction potential with equipotentials at $1 \mathrm{~V}$ steps showing clearly the low extraction field strength. In the model, the sample surface can be tilted and rotated to reflect the motion limits of the stage and to optimize the secondary ion collection efficiencies for both positive and negative ions. The actual arrangement in Fig. 1 that is modeled in Fig. 5(b) is far more complex than the opposing fields generated by the ideal geometry of Fig. 5(a). While most of the ions are attracted to the appropriate detector, this geometry results in some positive ions being repelled from the positive ion detector (with a similar result for negative ions).

\section{SUMMARY AND CONCLUSIONS}

A SIMS instrument with simultaneous positive and negative ion detection has been described, modeled, and some depth profiling results presented that illustrate its performance. Although a balanced system of opposing detectors is ideal, the nonideal case worked surprisingly well without compromising the performance specifications of either the SIMS detectors or the single-beam gallium ion microscope.

\section{ACKNOWLEDGMENTS}

The authors would like to acknowledge the diligence and recommendations of the reviewers in the final revision of this paper.

${ }^{1}$ P. H. Dawson and American Institute of Physics, Quadrupole Mass Spectrometry and Its Applications, American Vacuum Society Classics (American Institute of Physics, New York, 1995), p. 349.

${ }^{2}$ M. P. Ryan, D. E. Williams, R. J. Chater, B. M. Hutton, and D. S. McPhail, Nature 415, 770 (2002).

${ }^{3}$ H. Gnaser and H. Oechsner, Fresenius' J. Anal. Chem. 341, 54 (1991).

${ }^{4} \mathrm{~K}$. Wittmaack, "Surface and depth analysis based on sputtering," in Sputtering by Particle Bombardment III, edited by R. Behrisch and K. Wittmaack (Springer-Verlag, Berlin, 1991), pp. 161-256.

${ }^{5}$ K. Wittmaack, Int. J. Mass Spectrom. Ion Phys. 17, 39 (1975).

${ }^{6}$ H. Hayashi, T. Satake, K. Kaneko, and K. Nagai, Appl. Surf. Sci. 70-71, 287 (1993).

${ }^{7}$ K. Wittmaack, J. Maul, and F. Schulz, Int. J. Mass Spectrom. Ion Phys. 11, 23 (1973).

${ }^{8}$ S. Daolio, B. Facchin, C. Pagura, A. Tolstogouzov, and N. Konenkov, Rapid Commun. Mass Spectrom. 13, 782 (1999).

${ }^{9}$ R. J. Chater, B. A. Shollock, D. S. McPhail, A. J. Smith, and G. Cooke, Surf. Interface Anal. 46, 372 (2014).

${ }^{10}$ D. S. McPhail and M. G. Dowsett, "Dynamic SIMS," in Surface Analysis: The Principal Techniques, edited by J. C. Vickerman and I. S. Gilmore (John, Chichester, 2009), pp. 207-268.

11"FEI," FEI Company, Hillsboro, OR, accessed 25 February 2016, http:// www.feicompany.com.

12،"Hiden," Hiden Analytical Ltd., Warrington, accessed 25 February 2016, http://www.hidenanalytical.com.

${ }^{13}$ W. O. Hofer, "Angular, energy, and mass distribution of sputtered particles," in Sputtering by Particle Bombardment III, edited by R. Behrisch and K. Wittmaack (Springer-Verlag, Berlin, 1991), pp. 15-90.

${ }^{14}$ J. F. Ziegler, "SRIM2013 program download," accessed 25 February 2016, http://www.SRIM.org.

${ }^{15}$ P. Baertschi, Earth Planet. Sci. Lett 31, 341 (1976).

${ }^{16}$ R. A. De Souza, J. A. Kilner, and C. Jeynes, Solid State Ionics 97, 409 (1997).

${ }^{17}$ M. P. Seah, C. A. Clifford, F. M. Green, and I. S. Gilmore, Surf. Interface Anal. 37, 444 (2005).

18“"simion," Scientific Instrument Services, Inc., Ringoes, NJ, 08551-1054, see http://www.simion.com. 